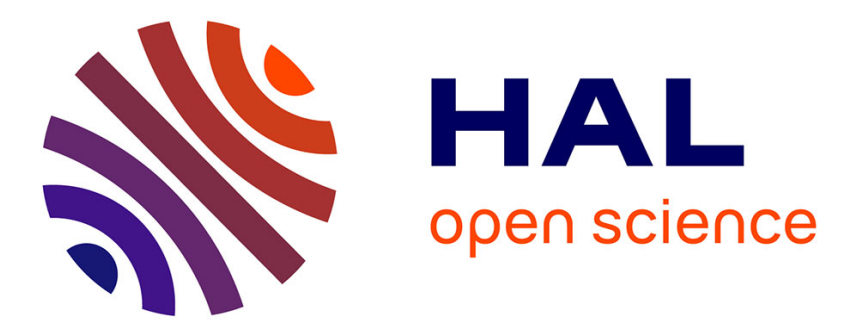

\title{
On computing estimates of the attraction region for a class of nonlinear time-delay systems
}

Daniel Melchor-Aguilar, Silviu-Iulian Niculescu

\section{To cite this version:}

Daniel Melchor-Aguilar, Silviu-Iulian Niculescu. On computing estimates of the attraction region for a class of nonlinear time-delay systems. 5th IFAC Symposium on Robust Control Design, ROCOND'06, Jul 2006, Toulouse, France. pp.626-631. hal-02294065

\section{HAL Id: hal-02294065 \\ https://hal.science/hal-02294065}

Submitted on 24 Sep 2019

HAL is a multi-disciplinary open access archive for the deposit and dissemination of scientific research documents, whether they are published or not. The documents may come from teaching and research institutions in France or abroad, or from public or private research centers.
L'archive ouverte pluridisciplinaire HAL, est destinée au dépôt et à la diffusion de documents scientifiques de niveau recherche, publiés ou non, émanant des établissements d'enseignement et de recherche français ou étrangers, des laboratoires publics ou privés. 


\title{
ON COMPUTING ESTIMATES OF THE ATTRACTION REGION FOR A CLASS OF NONLINEAR TIME-DELAY SYSTEMS
}

\author{
Daniel Melchor-Aguilar ${ }^{*, 1}$ Silviu-Iulian Niculescu ${ }^{* *}$
}

\author{
* Department of Applied Mathematics and Computer \\ Science,IPICYT, A.P.3-74,78216,San Luis Potosí,México \\ ** HEUDIASYC, Université de Technologie de Compiègne \\ Centre de Recherche de Royallieu, B.P. 20529, 60205, \\ Compiègne, France
}

\begin{abstract}
In this paper, initial estimates of the attraction region for a class of nonlinear time delay systems are proposed. The approach is constructive, and make use of an appropiate Lyapunov-Krasovskii functional. Several illustrative examples (delayed logistic equation, congestion networks, hereditary phenomena in physics) complete the paper.
\end{abstract}

Keywords: Nonlinear time-delay systems, attraction region, Lyapunov-Krasovskii functionals

\section{INTRODUCTION}

It is well-known from the theory of differentialdifference equations that if system

$$
\dot{x}(t)=A x(t)+B x(t-h),
$$

is exponentially stable (all zeros of its characteristic function have strictly negative real parts) then the trivial solution of the nonlinear system

$$
\dot{y}(t)=A y(t)+B y(t-h)+f(y(t), y(t-h)),
$$

where $f(u, v)$ satisfies a Lipschitz condition in a certain neigborhood of the origin and

$$
\lim _{\|(u, v)\| \rightarrow 0} \frac{\|f(u, v)\|}{\|(u, v)\|}=0,
$$

is asymptotically stable for sufficiently small initial conditions, see (Bellman and Cooke, 1963), (Halanay, 1966) and (Hale and Verduyn-Lunel, 1993). This result is the analogue of the PoincaréLyapunov theorem on stability with respect to the first approximation. The simpler method of

\footnotetext{
1 The corresponding author: dmelchor@ipicyt.edu.mx
}

proof is similar to the proof of the corresponding result for ordinary differential equations: by using the formula of variation of constants and the exponential upper bound for solutions of (1), see, e.g. (Bellman and Cooke, 1963), and (Hale and Verduyn-Lunel, 1993) for the general case of functional differential equations. As in the case of ordinary differential equations such method of proof does not provide us with a constructive estimate of the attraction region.

The result may be also proved by the method of Lyapunov-Krasovskii functionals. A stability theorem on the first approximation by using Lyapunov functionals was proved by A. Halanay in (Halanay, 1966). However, this does not also provide us with a constructive procedure to determine an initial estimate of the attraction region for a given nonlinear system (2).

The aim of this paper is to present a constructive method of computing initial estimates of the attraction region for the trivial solution of system (2) when the first approximation (1) is exponentially stable. 
The method we propose is analogue to the wellknown method of deriving initial estimates of the attraction region for systems $\dot{x}=A x+f(x)$ by means of quadratic Lyapunov functions. Thus, when the first approximation $\dot{x}=A x$ is exponentially stable, an initial estimate of the attraction region is given by the set $\left\{x \in \mathbb{R}^{n}: v(x)<c\right\}$, where $v(x)=x^{T} P x$ and $c>0$ is a constant depending on matrix $P$ and the nonlinear function $f(x)$, see for instance (Khalil, 1996). Here $P>0$ is the solution of the Lyapunov equation $A^{T} P+$ $P A=-W$ for any chosen $W>0$.

To the best of our knowledge, there is not a similar constructive method for delay systems of the form (2). A stability analysis for system (2), going from linear to nonlinear, by means of reduced Lyapunov functionals which are known to work in the linear stability analysis of system (1) is developed in (Verriest, 2000). As it is mentioned there the resulting stability conditions are in general difficult to check when matrix $B$ is not zero. For the case of matrix $B=0$, delay-indepedent estimates of the attraction region could be derived.

In our opinion the difficulties arising to construct quadratic Lyapunov-Krasovskii functionals associated to a given exponentially stable linear timedelay system seem to be the main reason for this lack. Some general expressions of quadratic functionals for linear time-delay systems have been proposed in (Huang, 1989), (Infante and Castelan, 1978) and (Repin, 1966). Whereas all these functionals are appropriate for stability analysis in the linear case, however, the form of the corresponding Lyapunov derivatives as well as the construction itself complicate the study in the robust and nonlinear cases. Recently, Kharitonov and Zhapko (Kharitonov and Zhabko, 2003) proposed a new class of Lyapunov-Krasovskii functionals which overcome such problems in the robust stability analysis case.

In this paper, the use of Lyapunov-Krasovskii functionals introduced in (Kharitonov and Zhabko, 2003) allows us to give a constructive initial estimate of the attraction region by using explicitly the functionals.

This paper is organized as follows: In section 2, after some preliminaries we present the construction of Lyapunov-Krasovskii functional for linear time delay systems according to (Kharitonov and Zhabko, 2003). In section 3 the main results are presented. The examples in section 4 illustrate the results, and some concluding remarks end the paper.

\section{PRELIMINARIES}

In this paper we consider time-delay systems of the following form

$$
\begin{aligned}
& \dot{y}(t)=A y(t)+B y(t-h)+f(y(t), y(t-h))(3) \\
& y(t)=\psi(t), t \in[-h, 0] .
\end{aligned}
$$

We assume that the initial function $\psi$ belongs to the space of continuous vector functions $\mathcal{C}=$ $\mathcal{C}\left([-h, 0], \mathbb{R}^{n}\right)$ equipped with the uniform norm $\|\psi\|_{h}=\sup _{\theta \in[-h, 0]}\|\psi(\theta)\|$, function $f(u, v)$ satisfies a Lipschitz condition in a certain neighborhood of the origin and

$$
\lim _{\|(u, v)\| \rightarrow 0} \frac{\|f(u, v)\|}{\|(u, v)\|}=0 .
$$

From these assumptions it holds that $f(0,0)=0$, and therefore system (3) has a trivial solution. We denote by $y_{t}(\psi)=\{y(t+\theta, \psi): \theta \in[-h, 0]\}$ the restriction of the solution $y(t, \psi)$ on the interval $[t-h, t]$. Throughout this paper we will use the Euclidean norm for vectors and the induced matrix norm for matrices, both denoted by $\|\cdot\|$.

Definition 1. The trivial solution of system (3) is stable if for any $\varepsilon>0$, there exists $\delta=\delta(\varepsilon)>0$ such that $\|\psi\|_{h}<\delta$ implies $\|y(t, \psi)\|<\varepsilon$ for $t \geq 0$.

Definition 2. The trivial solution of system (3) is asymptotic stable if it is stable and there exists $\delta_{a}>0$ such that $\|\psi\|_{h}<\delta_{a}$ implies $y(t, \psi) \rightarrow 0$ as $t \rightarrow \infty$.

Definition 3. The trivial solution of system (3) is exponentially stable if there exist constants $\delta_{e}>0, \mu \geq 1$ and $\alpha>0$ such that $\|\psi\|_{h}<\delta_{e}$ implies that $\|y(t, \psi)\| \leq \mu\|\varphi\|_{h} e^{-\alpha t}, t \geq 0$.

Now we present the construction procedure of quadratic Lyapunov functionals for linear timedelay systems in the spirit of (Kharitonov and Zhabko, 2003).

Consider the following linear time-delay system

$$
\begin{aligned}
& \dot{x}(t)=A x(t)+B x(t-h) \\
& x(t)=\varphi(t), t \in[-h, 0] .
\end{aligned}
$$

Assume that system (5) is exponentially stable, i.e., for any $\varphi \in \mathcal{C}$ there exist constants $\mu \geq 1$ and $\alpha>0$ such that $\|x(t, \varphi)\| \leq \mu\|\varphi\|_{h} e^{-\alpha t}, t \geq 0$.

Selecting positive definite matrices $W_{j}, j=0,1,2$ consider the following functional

$$
\begin{aligned}
& w(\varphi)=\varphi^{T}(0) W_{0} \varphi(t)+\varphi^{T}(-h) W_{1} \varphi(-h)(6) \\
& +\int_{-h}^{0} \varphi^{T}(\theta) W_{2} \varphi(\theta) d \theta,
\end{aligned}
$$

where $\varphi \in \mathcal{C}$ is arbitrary. If system (5) is exponentially stable, then there exists a unique quadratic 
functional $v: \mathcal{C} \mapsto \mathbb{R}$ such that $t \mapsto v\left(x_{t}(\varphi)\right)$ is differentiable for $t \geq 0$, and

$$
\frac{d v\left(x_{t}(\varphi)\right)}{d t}=-w\left(x_{t}(\varphi)\right), t \geq 0
$$

for all solutions $x(t, \varphi)$ of $(5)$. Functional $v(\cdot)$ is called the Lyapunov-Krasovskii functional associated with (5) (Kharitonov and Zhabko, 2003). The Lyapunov-Krasovskii functional is of the form

$$
\begin{aligned}
& v(\varphi)=\varphi^{T}(0) U(0) \varphi(0) \\
& -2 \varphi^{T}(0) \int_{-h}^{0} U(-h-\theta) B \varphi(\theta) d \theta \\
& +\int_{-h}^{0} \int_{-h}^{0} \varphi^{T}\left(\theta_{1}\right) B^{T} U\left(\theta_{1}-\theta_{2}\right) B \varphi\left(\theta_{2}\right) d \theta_{1} d \theta_{2} \\
& +\int_{-h}^{0} \varphi^{T}(\theta)\left(W_{1}+(h+\theta) W_{2}\right) \varphi(\theta) d \theta
\end{aligned}
$$

where the matrix function $U(\cdot)$ is defined as

$$
U(\tau)=\int_{0}^{\infty} K^{T}(t) W K(t+\tau) d t, \tau \in[-h, h],
$$

where $W=W_{0}+W_{1}+h W_{2}, K(t)$ is the fundamental matrix of $(5)$, i.e., $K(t)$ is the unique matrix function which satisfies

$$
\dot{K}(t)=A K(t)+B K(t-h), t>0,
$$

with the initial condition $K(t)=0$ for all $t<0$, and $K(0)=I$, see (Bellman and Cooke, 1963).

Note that by the exponential stability of (5) the matrix $U(\tau)$ is well defined. The matrix function $U(\cdot)$ satisfies the following second-order ordinary differential equation, see (Huang, 1989) and (Kharitonov and Zhabko, 2003),

$\ddot{U}(\tau)=\dot{U}(\tau) A-A^{T} \dot{U}(\tau)+A^{T} U(\tau) A-B^{T} U(\tau) B$,

with the following additional conditions

$$
\begin{aligned}
\dot{U}(0) & =U(0) A+U^{T}(h) B \\
-W & =\dot{U}(0)+(\dot{U}(0))^{T}
\end{aligned}
$$

Remark 1. When system (5) is exponentially stable the matrix function (8) is the unique solution of (9) satisfying (10) and (11), see (Kharitonov and Plischke, 2004). A piece-wise linear approximation of matrix function $U(\cdot)$ can be computed from equations (9)-(11) (Kharitonov and Garcia, 2004). In the scalar case is possible to obtain an explicit solution of the equations (9)-(11), see (Melchor-Aguilar, 2004).

Proposition 1. Let system (5) be exponentially stable. Given any $W_{j}>0, j=0,1,2$, the functional (7) satisfies:

(1) $\alpha_{1}\|\varphi(0)\|^{2} \leq v(\varphi) \leq \alpha_{2}\|\varphi\|_{h}^{2}$, for some constants $\alpha_{1}>0$ and $\alpha_{2}>0$,

(2) $\frac{d}{d t} v\left(x_{t}(\varphi)\right) \leq-\beta\|x(t, \varphi)\|^{2}$, for a constant $\beta>0$.
Indeed, the lower bound of $v(\varphi)$ holds for

$$
\alpha_{1}<\min \left\{\frac{\lambda_{\min }\left(W_{0}\right)}{2\|A\|+\|B\|}, \frac{\lambda_{\min }\left(W_{1}\right)}{\|B\|}\right\} .
$$

Let

$$
u_{0}=\max _{\tau \in[0, h]}\|U(\tau)\|
$$

Functional $v(\varphi)$ satisfies

$$
v(\varphi) \leq \kappa\left(\|\varphi(0)\|^{2}+\int_{-h}^{0}\|\varphi(\theta)\|^{2} d \theta\right),
$$

where

$$
\begin{aligned}
\kappa= & \max \left\{u_{0}(1+h\|B\|)\right. \\
& \left.u_{0}\|B\|(1+h\|B\|)+\left\|W_{1}+h W_{2}\right\|\right\} .
\end{aligned}
$$

Then

$$
v(\varphi) \leq \alpha_{2}\|\varphi\|_{h}^{2}, \text { for } \alpha_{2} \geq \kappa(1+h) .
$$

\section{MAIN RESULTS}

In this section the main results of the paper are presented. We show that an initial estimate of the attraction region for the trivial solution of (3) can be computed when the first approximation (5) is exponentially stable.

From (4) follows that for any $\gamma>0$ there exists $\delta(\gamma)>0$ such that if $\|(y(t), y(t-h))\|<\delta$ then

$$
\|f(y(t), y(t-h))\|<\gamma\|(y(t), y(t-h))\| .
$$

Theorem 2. Let system (5) be exponentially stable. For

$$
\begin{aligned}
\gamma< & \min \left\{\frac{\lambda_{\min }\left(W_{0}\right)}{u_{0}(2+\|B\| h)},\right. \\
& \left.\frac{\lambda_{\min }\left(W_{1}\right)}{u_{0}(1+\|B\| h)}, \frac{\lambda_{\min }\left(W_{2}\right)}{\|B\| u_{0}}\right\},
\end{aligned}
$$

the set

$$
\mathcal{U}=\left\{\psi \in \mathcal{C}: v(\psi)<\alpha_{1} \frac{\delta^{2}}{4} \text { and }\|\psi\|_{h}<\frac{\delta}{2}\right\}
$$

is an estimate of the attraction region for the trivial solution of system (3).

Corollary 3. Let system (5) be exponentially stable. For any solution $y(t, \psi)$ of (3) such that $\psi \in \mathcal{U}$, the following exponential estimate holds:

$$
\|y(t, \psi)\| \leq \sqrt{\frac{\alpha_{2}}{\alpha_{1}}}\|\psi\|_{h} e^{-\frac{\beta}{2 \kappa} t}, t \geq 0,
$$

where

$$
\begin{gathered}
\beta=\min \left\{\lambda_{\min }\left(W_{0}\right)-\gamma u_{0}(2+\|B\| h)\right. \\
\left.\lambda_{\min }\left(W_{2}\right)-\|B\| u_{0} \gamma\right\},
\end{gathered}
$$


Hence, according with definition 3 the trivial solution of (3) is exponentially stable.

Note that, by combining $\mathcal{U}$ with the upper bound for functional $v(\psi)$ we can obtain the following more conservative, yet computational more tractable, estimate of the attraction region:

$$
\mathcal{V}=\left\{\psi \in \mathcal{C}:\|\psi\|_{h}<\sqrt{\frac{\alpha_{1}}{\alpha_{2}}} \delta\right\} \subseteq \mathcal{U}
$$

Remark 2. As in the case of system without delay, estimating the region of attraction by means of the set $\mathcal{U}$ is simple but usually conservative. Clearly, the estimates of the attraction region (15) and (17) depend on the choice of the matrices $W_{j}, j=0,1,2$. These matrices can be used as free parameters in order to optimize such estimates.

\section{EXAMPLES}

In this section we illustrate our results by some examples from the literature.

\subsection{Delayed Logistic Equation}

Let us consider the following delayed logistic equation

$$
\dot{x}(t)=x(t)\left[r-m x(t)-n x^{2}(t-h)\right] .
$$

Equation (18) is a generalization of the so-called delayed logistic equation encountered in several problems in biological systems (population dynamics, single species growth model, etc.) see (Gopalsamy, 1992). Equation (18) has a unique positive equilibrium point defined by

$$
x^{*}=\frac{-m+\sqrt{m^{2}+4 r n}}{2 n} .
$$

Let $y(t)=x(t)-x^{*}$, then

$$
\dot{y}(t)=a y(t)+b y(t-h)+f(y(t), y(t-h)),
$$

where $a=-m x^{*}, b=-2 n\left(x^{*}\right)^{2}$ and

$$
f(u, v)=-m u^{2}-2 n x^{*} u v-n x^{*} v^{2}-n u v^{2} .
$$

We have

$$
\begin{aligned}
|f(u, v)| \leq & m u^{2}+n x^{*}\left(u^{2}+v^{2}\right)+n x^{*} v^{2} \\
& +(n / 2)\left(u^{2}+v^{2}\right)^{\frac{3}{2}} .
\end{aligned}
$$

Let $\varsigma=\max \left\{m, n x^{*}\right\}$, then

$$
|f(u, v)| \leq 2 \varsigma\left(u^{2}+v^{2}\right)+(n / 2)\left(u^{2}+v^{2}\right)^{\frac{3}{2}} .
$$

Thus, for any given $\gamma>0$ there exists $\delta(\gamma)>0$, as the unique real positive solution of the equation $2 \varsigma \delta+(n / 2) \delta^{2}=\gamma$, such that $|f(u, v)|<\gamma\|(u, v)\|$ if $\|(u, v)\|<\delta$. The first approximation

$$
\dot{x}(t)=a x(t)+b x(t-h),
$$

is exponentially stable for $b<a$ if and only if $h \in\left[0, h_{0}\right)$ where

$$
h_{0}=\frac{\arccos \left(-\frac{a}{b}\right)}{\sqrt{b^{2}-a^{2}}} .
$$

In (Gopalsamy, 1992) this fact is used to conclude, by means of a direct application of the stability theorem with respect to the first approximation, that the trivial solution of (19) is asymptotically stable for $h \in\left[0, h_{0}\right)$ in a sufficiently small neighborhood of the origin, and then to investigate the existence of periodic solutions of (19) for $h$ near $h_{0}$.

Here we show how the application of our results can provide an initial estimate of such neighborhood (the attraction region) for the trivial solution of (19). In this case equations (9)-(11) look as

$$
\begin{aligned}
\ddot{U}(\tau) & =\left(a^{2}-b^{2}\right) U(\tau), \\
\dot{U}(0) & =a U(0)+b U(h), \\
-W & =2 \dot{U}(0) .
\end{aligned}
$$

If equation (20) is exponentially stable, the unique solution of (21) satisfying (22) and (23), see (Melchor-Aguilar, 2004), is $U(\tau)=W u(\tau), \tau \in$ $[0, h]$, where

$u(\tau)=\left(\frac{-\lambda+b \sin (\lambda h)}{2 \lambda(a+b \cos (\lambda h))}\right) \cos (\lambda \tau)-\frac{1}{2 \lambda} \sin (\lambda \tau)$, where $\lambda=\sqrt{b^{2}-a^{2}}$. As an example consider in (18) that $r=1, m=1$ and $n=2$, then the unique positive equilibrium point of (18) is $x^{*}=0.5$. Thus we get $a=-0.5, b=-1$, and $h_{0}=2.4184$. So, taking $h=1$ we have that (20) is exponentially stable.

Let $W_{0}=1.5, W_{1}=1$ and $W_{2}=0.5$, then $u_{0}=2.4562$. Direct calculations derived from (14) show that $\gamma<0.2036$. Selecting $\gamma=0.2035$ we obtain $\delta=0.0970$. From (12) and (13) we get $\alpha_{1}<0.75$ and $\alpha_{2} \geq 12.8249$. Selecting $\alpha_{1}=0.74$ and $\alpha_{2}=12.8249$, the estimates of the attraction region for the trivial solution of (19), defined by (15) and (17) are given by

$\mathcal{U}=\left\{\psi \in \mathcal{C}: v(\psi)<0.0017\right.$ and $\left.\|\psi\|_{1}<0.0485\right\}$, and

$$
\mathcal{V}=\left\{\psi \in \mathcal{C}:\|\psi\|_{1}<0.0117\right\} .
$$

From (16) we obtain the following exponential bound for any solution starting in the set $\mathcal{U}$

$$
\|y(t, \psi)\| \leq 4.1630\|\psi\|_{1} e^{-1.225 \times 10^{-5} t}, t \geq 0 .
$$

Now we illustrate the dependence of the attration region on the delay value. Let us select $h=0.5$, then (20) is exponentially stable. Let now $W_{0}=$ 1.5, $W_{1}=0.9$ and $W_{2}=0.6$, then $u_{0}=1.4237$. From (14) we obtain $\gamma<0.4214$. Choosing $\gamma=$ 0.4213 we get $\delta=0.1922$. From (12) and (13) 
we obtain $\alpha_{1}<0.75$ and $\alpha_{2} \geq 5.0032$. Selecting $\alpha_{1}=0.74$ and $\alpha_{2}=5.0032$, the estimates of the attraction region (15) and (17) are given by

$\mathcal{U}=\left\{\psi \in \mathcal{C}: v(\psi)<0.0068\right.$ and $\left.\|\psi\|_{0.5}<0.0961\right\}$, and

$$
\mathcal{V}=\left\{\psi \in \mathcal{C}:\|\psi\|_{0.5}<0.0370\right\} .
$$

The corresponding exponential bound is given by

$$
\|y(t, \psi)\| \leq 2.6\|\psi\|_{0.5} e^{-3.1391 \times 10^{-5} t}, t \geq 0 .
$$

\subsection{Congestion control problem in networks}

Let us consider the following nonlinear equation

$$
\dot{x}(t)=k[w-x(t-h) p(x(t-h))] .
$$

where $k, w$ are positive reals and $p(\cdot)$ is a continuous and differentiable nondecreasing function. Equation (26) describes the dynamics of a collection of flows, all using a single resource, and sharing the same gain parameter $k$, see (Kelly, 2000). The delay $h$ represents the round-trip time, and is assumed constant. The function $p(\cdot)$ can be interpreted as the fraction of packets indicating (potential) congestion (presence), see (Kelly, 2000).

Considering that function $p(x)=k_{p} x$ with $k_{p}>0$, (see (Hollot-et-al, 2002) for a discussion on the benefits to use a proportional controller) equation (27) can be written as

$$
\dot{x}(t)=k\left(w-k_{p} x^{2}(t-h)\right),
$$

The unique positive equilibrium point of $(27)$ is $x^{*}=\sqrt{\frac{w}{k_{p}}}$. Let $y(t)=x(t)-x^{*}$, then

$$
\dot{y}(t)=b y(t-h)+f(y(t-h)) .
$$

where $b=-2 k k_{p} x^{*}$ and $f(y(t-h))=-k k_{p} y^{2}(t-$ $h)$. For any given $\gamma>0$ there exists $\delta=\frac{\gamma}{k k_{p}}$ such that $|f(y(t-h))|<\gamma|y(t-h)|$ if $|y(t-h)|<\delta$.

The first approximation

$$
\dot{y}(t)=b y(t-h),
$$

is exponentially stable if and only

$$
2 b h>-\pi \text { or } k_{p}<\frac{\pi^{2}}{16 w(h k)^{2}} .
$$

In this case, equations (9)-(11) look as

$$
\begin{aligned}
\ddot{U}(\tau) & =-b^{2} U(\tau), \\
\dot{U}(0) & =b U(h), \\
2 \dot{U}(0) & =-W .
\end{aligned}
$$

If equation (29) is exponentially stable, then the unique solution of (30) satisfying (31) and (32), see (Melchor-Aguilar, 2004), is $U(\tau)=W u(\tau)$, $\tau \in[0, h]$, where

$$
u(\tau)=\left(\frac{-1+\sin (b h)}{2 b \cos (b h)}\right) \cos (b \tau)-\frac{1}{2 b} \sin (b \tau) .
$$

As an example consider the network parameters from (Kunniyur and Srikant, 2003): $k=0.01$, $w=1$, and $h=0.1$. Taking $k_{p}=0.01$ we obtain $x^{*}=10$. Thus, equation (29) is exponentially stable.

Let $W_{0}=1, W_{1}=0.5$ and $W_{2}=0.1$, then we have $u_{0}=377.5755$. Direct calculations derived from (14) show that $\gamma<0.013$. Selecting $\gamma=0.012$ we obtain $\delta=12$. From (12) and (13) we get $\alpha_{1}<250$ and $\alpha_{2} \geq 415.4161$. Selecting $\alpha_{1}=249$ and $\alpha_{2}=415.4161$, the estimates of the attraction region for the trivial solution of (28) defined by (15) and (17) are given by

$$
\mathcal{U}=\left\{\psi \in \mathcal{C}: v(\psi)<8964 \text { and }\|\psi\|_{0.1}<6\right\},
$$

and

$$
\mathcal{V}=\left\{\psi \in \mathcal{C}:\|\psi\|_{0.1}<7.7498\right\}
$$

The corresponding exponential bound (16) for any solution starting in the set $\mathcal{U}$ is given by

$$
\|y(t, \psi)\| \leq 1.2916\|\psi\|_{0.1} e^{-1.2409 \times 10^{-4} t}, t \geq 0 .
$$

\subsection{Hereditary phenomena in physic}

Let us consider the following equation

$$
\ddot{x}(t)+2 r \dot{x}(t)+p^{2} x(t)+2 q \dot{x}(t-1)=\varepsilon \dot{x}^{3}(t-1) .
$$

Equation (33) arises in modelling the dynamics of oscillations in a vacuum tube, in the theory of self-excited oscillations, and in the theory of stabilization of ships, see (Kolmanovskii and Myshkis, 1999) and the references therein.

Let $y_{1}(t)=x(t)$ and $y_{2}(t)=\dot{x}(t)$, then $(33)$ can be written as

$$
\dot{y}(t)=A y(t)+B y(t-1)+f(y(t-1)),
$$

where $y(t)=\left(\begin{array}{ll}y_{1}(t) & y_{2}(t)\end{array}\right)^{T}, A=\left(\begin{array}{cc}0 & 1 \\ -p^{2} & -2 r\end{array}\right)$, $B=\left(\begin{array}{cc}0 & 0 \\ 0 & -2 q\end{array}\right)$ and $f(y(t-1))=\left(\begin{array}{lll}0 & \varepsilon y_{2}^{3}(t-1)\end{array}\right)^{T}$.

The unique equilibrium point of (34) is the origin. For any given $\gamma>0$ there exists $\delta=\sqrt{\frac{\gamma}{\varepsilon}}$ such that

$$
\|f(y(t-1))\|<\gamma\|y(t-1)\| \text { if }\|y(t-1)\|<\delta .
$$

The first approximation of (34) is

$$
\dot{y}(t)=A y(t)+B y(t-1) .
$$

Applying a frequency sweeping test it is easy to show that (35) is delay-independent exponentially stable if $2\left(r^{2}+q^{2}\right)>p^{2}$, see (Niculescu, 2001). Thus, considering $r=p=q=1$ we have that (35) is exponentially stable.

Let $W_{0}=I, W_{1}=0.75 I$ and $W_{2}=0.5 I$. A piecewise linear approximation of the unique matrix $U(\cdot)$ solution of equations (9)-(11) is plotted in Fig. 1. 


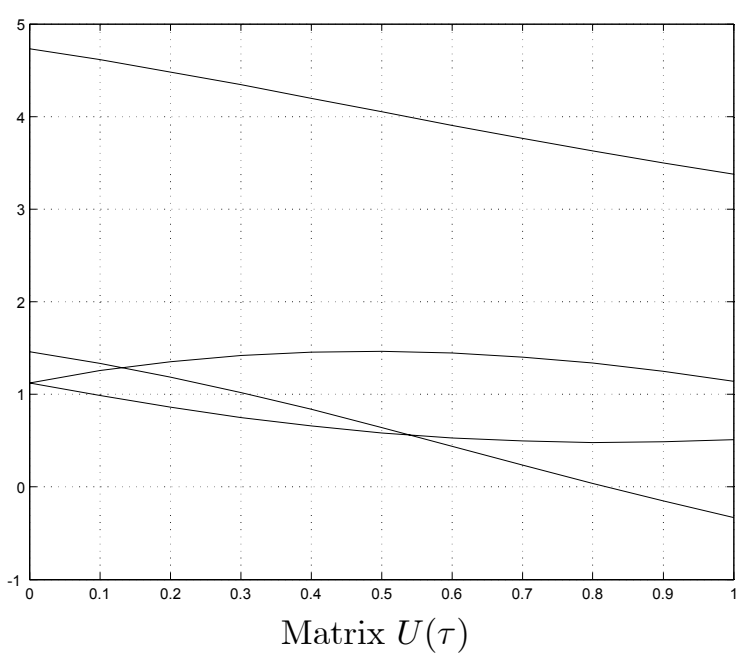

From Fig. 1 we have that $u_{0}=5.0801$. Direct calculations derived from (14) show that $\gamma<$ 0.0492. Selecting $\gamma=0.049$ and considering $\varepsilon=$ 0.5 we obtain $\delta=0.3130$.. From (12) and (13) we get $\alpha_{1}<0.1464$ and $\alpha_{2} \geq 63.4618$. Taking $\alpha_{1}=0.1463$ and $\alpha_{2}=63.4618$ the estimates of the attraction region defined by (15) and (17) are given by

$\mathcal{U}=\left\{\psi \in \mathcal{C}: v(\psi)<0.0036\right.$ and $\left.\|\psi\|_{1}<0.1565\right\}$, and

$$
\mathcal{V}=\left\{\psi \in \mathcal{C}:\|\psi\|_{1}<0.0075\right\} .
$$

The corresponding exponential upper bound (16) for any solution of (34) starting in the set $\mathcal{U}$ is

$$
\|y(t, \psi)\| \leq 20.8487\|\psi\|_{h} e^{-3.3808 \times 10^{-5} t}, t \geq 0 .
$$

\section{CONCLUSION}

A constructive procedure to compute initial estimates of the attraction region for a class of nonlinear time-delay systems whenever the first approximation is exponentially stable is proposed. The approach is constructive and make use of the Lyapunov-Krasovskii functional associated to the first approximation. Some examples illustrate the results.

\section{REFERENCES}

Bellman R. and Cooke K. L., Differential and difference equations, Academic Press, New York, 1963.

Halanay A., Differential equations: Stability Oscillations and time lags, Academic Press, New York, 1966.

Hale J. K. and Verduyn Lunel S. M., Introduction to functional differential equations, Springer Verlag, New York, 1993.

Gopalsamy K., Stability and Oscillations in Delay Differential Equations of Population Dynamics, Math. Its Appl. Series, 74, Kluwer Academic Publishers, 1992.
Hollot C.V., Misra V., Towsley D., Gong W., "Analysis and design of controllers for AQM routers supporting TCP flows", IEEE Trans. Automatic Control, 47, No. 6, 2002.

Huang Y. S., "Generalization of the Lyapunov's theorem in a linear delay system", J. Math. Anal. Appl., 182, 83-94, 1989.

Infante E. F. and Castelan W. B., "A Lypunov functional for a matrix difference differential equation", J. Differential Eqns, 29, 439-451, 1978.

Kelly F. P, "Mathematical modelling of the internet", in Mathematics unlimited - 2001 and beyond (Eds. B. Engquist, W. Schmid), SpringerVerlag: Berlin, 685-702.

Khalil, H. K., Nonlinear systems, Prentice Hall, 1996.

Kharitonov V.L. and Zhabko A.P., "LyapunovKrasovskii approach to robust stability of time delay systems", Automatica, 39, 15-20, 2003

Kharitonov V.L. and Plischke E., "Lyapunov matrices for time-delay systems", Report 0405, Zentrum für Technomathematik, Universität Bremen, ISSN 1435-7968, April 2004.

Kharitonov V.L. and Garcia-Lozano H., "Lyapunov matrices for time-delay systems with commesurate delays", in Proceeding of IFAC Symposium on System Structure and Control, Oaxaca, México, 2004.

Kolmanovskii V. and Myshkis A., Introduction to the theory and applications of functional differential equations, Kluwer Academic Publishers, 1999.

Kunniyur S. and Srikant R., "Stable, Scalable, Fair Congestion Control and AQM Schemes that Achive High Utilization in the Internet", IEEE Trans. Automatic Control, 48, No. 11, 2003.

Melchor Aguilar D., "On the Lyapunov's indirect method for scalar differential-difference equations", in Proceeding of IFAC Symposium on System Structure and Control, Oaxaca, México, 2004.

Niculescu, S.-I., Delay Effects on Stability: A Robust Control Approach, Springer-Verlag: Heidelberg, LNCIS, vol. 269, 2001.

Repin I. M., "Quadratic Lyapunov functionals for systems with delay", J. Appl. Math. Mech., 29, 669-672, 1966. Tranlation of Prikl. Mat. Meh., 29:564-566 (1965).

Verriest E.I., "Robust Stability and Stabilization: From Linear to Nonlinear", in Proceeding of $2 n d$ IFAC Workshop on Linear Time Delay Systems, Ancona, Italy, 2000. 